Klaudiusz A. Nadolny ${ }^{1,2}$, Dorota Zyśko ${ }^{3}$, Dariusz Boroń ${ }^{1}$,Jerzy R. Ładny ${ }^{4}$, Robert Gałązkowski ${ }^{5}$, Kamil Bujak ${ }^{6}$, Mariusz Gąsior ${ }^{6}$, Jacek Kubica ${ }^{7}$, Artur Fedorowski ${ }^{8}, 9$, Richard Sutton ${ }^{8}, 10$

${ }^{1}$ Faculty of Medicine, Katowice School of Technology, Katowice, Poland

2Department of Health Sciences, WSB University, Dabrowa Gornicza, Poland

${ }^{3}$ Department of Emergency Medicine, Wroclaw Medical University, Wroclaw, Poland

${ }^{4}$ Department of Emergency Medicine, Medical University of Bialystok, Poland

${ }^{5}$ Department of Emergency Medical Service, Medical University of Warsaw, Poland

${ }^{6} 3^{\text {rd }}$ Department of Cardiology, Silesian Center for Heart Diseases, Faculty of Medical Sciences in Zabrze, Medical University of Silesia,

Katowice, Poland

${ }^{7}$ Department of Cardiology and Internal Medicine, Collegium Medicum, Nicolaus Copernicus University, Bydgoszcz, Poland

${ }^{8}$ Department of Clinical Sciences, Lund University, Clinical Research Center, Malmo, Sweden

${ }^{9}$ Department of Cardiology, Skane University Hospital, Malmo, Sweden

${ }^{10}$ National Heart and Lung Institute, Imperial College, Hammersmith Hospital Campus, Ducane Road, London, United Kingdom

\title{
Does the position of the body impact the return of spontaneous circulation and hospital survival in sudden cardiac arrest patients?
}

\section{Corresponding author:}

Klaudiusz Nadolny, Faculty of Medicine Katowice School of Technology, Rolna 43 Str., 40-555 Katowice, Poland e-mail: prrm.knadolny@interia.pl
Medical Research Journal 2022; Volume 7, Number 1, 10-16 DOI: 10.5603/MRJ.a2022.0003 Copyright (C) 2022 Via Medica

ISSN 2451-2591 e-ISSN 2451-4101

\begin{abstract}
Introduction: Out-of-hospital cardiac arrest (OHCA) is a big medical problem.

Material and methods: The aim of this study was to assess the occurrence of sitting position in victims of $\mathrm{OHCA}$ at the arrival of Emergency Medical Services (EMS) and examine whether maintaining a supine position until ambulance arrival impacts the return of spontaneous circulation (ROSC) and survival until hospital admission and discharge. The documentation of patients in the OHCA registry of part of Silesian Voivodeship 1-06.2018 was accessed and yielded 634 cases for analysis.

Results: The sitting position was found in 41 (6.5\%) patients. These patients were more frequently female: $46.3 \%$ vs $30.6 \%$, and older: 74.0 (57.9-82.0) vs 67.7 (54.9-75.8) and less frequently received cardiopulmonary resuscitation (CPR) from bystanders: $7.3 \%$ vs 63.6\%, while ROSC was found with similar frequency: $34.2 \%$ vs $34.1 \%$, survival until hospital admission in ROSC subgroups was lower in those in a sitting position than in a lying position: $50 \%$ vs $75.5 \%$. None patients who were found in the sitting position survived until hospital discharge, while $33(6.1 \%)$ patients out of those found in the lying positions survived. The univariable analysis showed that the sitting position was associated with female sex, older age, previously implanted implantable cardioverter-defibrillator (ICD) before OHCA. The multivariable logistic revealed that the sitting position was independently associated with chest pain and the presence of ICD.

Conclusions: Patients found in a sitting position at the arrival of EMS constituted $6.5 \%$ of patients with OHCA in whom resuscitation attempts were undertaken and were at higher risk of unfavorable outcomes. Key words: cardiac arrest, cardiopulmonary resuscitation, sitting position
\end{abstract}

Med Res J 2022; 7 (1): 10-16

\section{Introduction}

Cardiac arrest is a cessation of cardiac mechanical function leading to loss of consciousness, apnoea or stertorous breathing, and impalpable large arterial pulse [1] Depending on how out-of-hospital cardiac arrest (OHCA) is defined, the condition is diagnosed in Europe with the incidence of 38/100,000 inhabitants per year [2,3], in the United States - 76/100,000 inhabitants per year [4], and in Poland - 69.7-95.0/100 000 inhabitants per year [5, 6].

The majority of cardiac arrest victims are in a lying position, in which they may be at the onset of cardiac 
arrest or which they assume as a result of falling due to a decrease in postural muscle tone [7, 8]. Patients who are initially in a seated position usually fall down unless supported by people or objects in the environment or if they have a low center of gravity $[9,10]$.

The position of a patient's body during cardiac arrest may delay the correct diagnosis and resuscitation by witnesses or even the members of medical services $[10,11]$.

The importance of a maintained sitting position during a cardiac arrest for the recognition of the condition and its' prognosis is unknown. The sitting position may be accidental or caused by the adoption of this position before the arrest due to premonitory symptoms. However, it cannot be ruled out that sitting may be associated with activities involved in the cause of cardiac arrest, such as passing urine or stool [12]. Delay in assuming a horizontal position is likely to extend the duration of loss of consciousness (LOC) and potentially lead to permanent cardiac arrest [13]. Moreover, the defibrillation threshold is likely to be rising at this time leading to ineffective shocks [14].

The aim of this study was to assess the occurrence of sitting position in victims of OHCA at the arrival of Emergency Medical Services and examine whether maintaining a supine position until ambulance arrival impacts the return of spontaneous circulation (ROSC) and survival until hospital admission and discharge in patients who received cardiopulmonary resuscitation (CPR).

\section{Material and methods}

The study group and methods were described elsewhere [15]. A brief questionnaire regarding cardiac arrest data and circumstances was completed by Emergency Medical Services (EMS) personnel in upper region Poland. In Poland, EMS can perform endotracheal intubation and administer drugs according to the guidelines for resuscitation or defibrillation manual, etc. The Silesian Registry of Out-of-Hospital Cardiac Arrest (SIL-OHCA; Clinical Trials. Gov Identifier: NCT03654859) provided the study material. From a total of 853 questionnaires compiled between January and June 2018, the following questionnaires were excluded: 162 cases where the cardiac arrest occurred after EMS arrival, one questionnaire concerning a patient referred to Helicopter Emergency Medical Services (HEMS), 42 questionnaires covering the same patients completed by first responders and specialist EMS personnel, and 14 questionnaires concerning patients in whom the position of the body at EMS arrival was classified as other than sitting or lying, or was not assessed, yielding 634 cases qualifying for inclusion in the study.

The following data were gathered based on the questionnaire: sex, age, place of incident, assessment of the mechanism of cardiac arrest as traumatic or non-traumatic, presence of witnesses of the event, bystander CPR consisting of automated external defibrillation (AED) or chest compressions, symptoms before cardiac arrest (chest pain, dyspnoea, weakness), comorbidities (previous myocardial infarction, diabetes, previous stroke, coronary artery bypass graft [CABG]/ percutaneous coronary interventions [PCI], pacemaker [PM], implantable cardioverter-defibrillator [ICD]) if available to bystanders, duration between the call and EMS arrival, body position at EMS arrival, first recorded rhythm, defibrillation, the airways management, and vascular access, prehospital ROSC, and survival until hospital admission and discharge.

The data on in-hospital mortality rates were provided by the National Health Fund (NHF), the sole public provider of healthcare services in Poland; the data were available for 580 patients.

The number and percentage of patients with missing data on characteristics, the event setting, performed procedures, and outcomes are shown in the supplementary materials (Tab. S1).

ROSC was defined as any documented return of adequate circulation achieved during the prehospital phase during the event. Survival until hospital admission was defined as a transfer of care to the medical staff at the receiving hospital, independent of the patient's ROSC status (with sustained ROSC or during ongoing CPR).

The Bioethical Commission of Silesian Medical Chamber assessed that an opinion of the Bioethical Commission is not necessary for collecting data from The Silesian Registry of Out-of-Hospital Cardiac Arrest.

\section{Statistical analysis}

Continuous data are presented as the median and interquartile range (IQR) and compared using a Mann-Whitney $U$ test or numbers and percentages and compared using a chi ${ }^{2}$ test.

Univariable and multivariable logistic regression analyses were performed to find factors associated with the sitting position and survival until hospital admission, which was the study's primary endpoint. The univariable analysis of factors associated with sitting position included sex, age, OHCA aetiology, arrest location, witnesses of the event, previous myocardial infarction, previous stroke, a previous pacemaker, or implantable cardioverter-defibrillator, dyspnoea or chest pain before cardiac arrest, and shockable rhythm. The univariable model regarding survival until hospital admission additionally encompassed the following factors: sitting position, adrenaline administration, and endotracheal intubation. All variables significantly associated with the outcome in the univariable analysis were included in the multivariable model. 


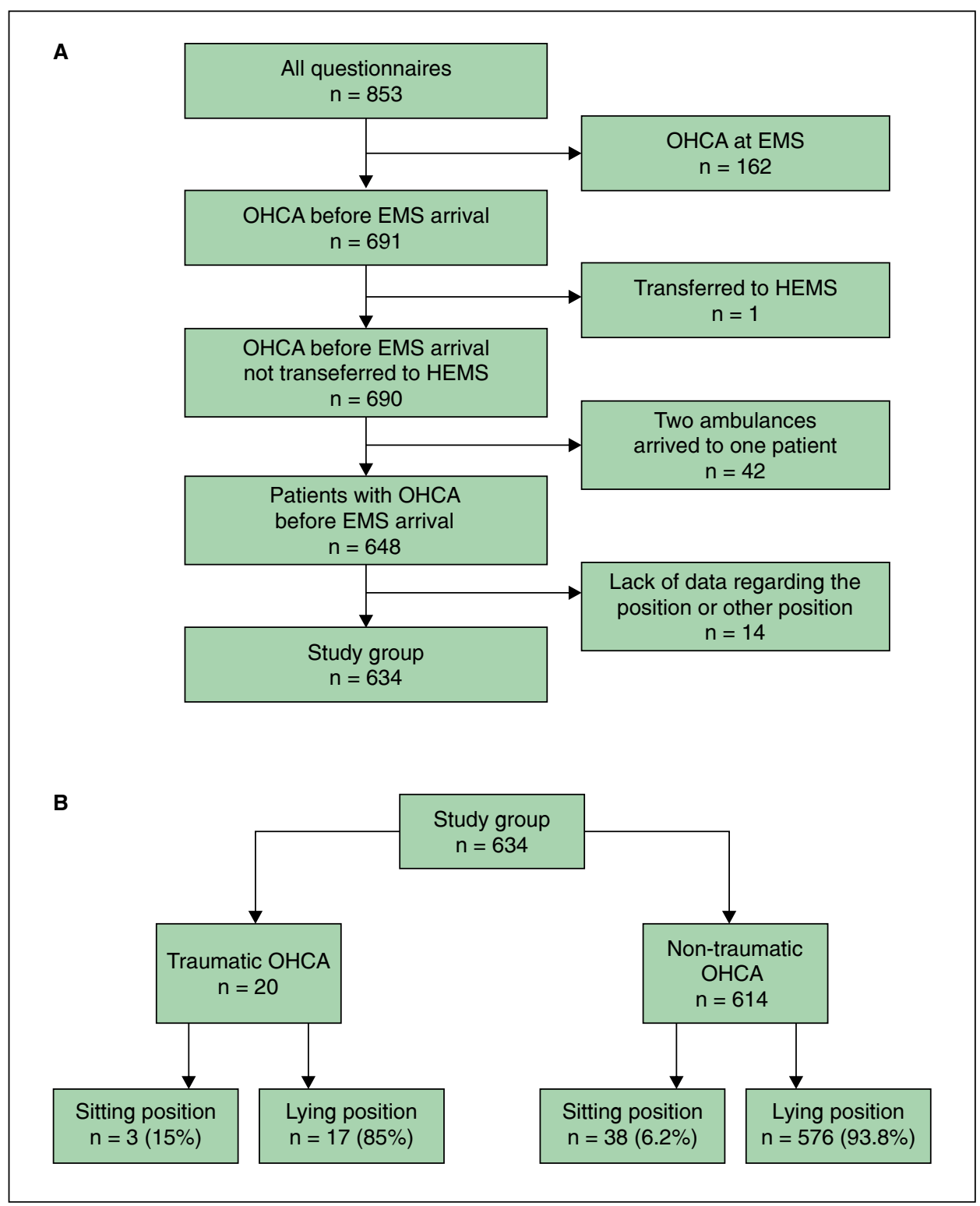

Figure 1. A. Flow chart. Study group construction; B. Patients with traumatic and non-traumatic cardiac arrest found in a sitting position

The Bioethical Commission of Silesian Medical Chamber decided that an opinion of the Bioethical Commission is not necessary for collecting data from The Silesian Registry of Out-of-Hospital Cardiac Arrest. The present study was performed on the basis of the above Registry. The study follows the principles of the Declaration of Helsinki. $\mathrm{P}<0.05$ was considered significant. Statistica version 13.3 (TIBCO Software, CA, USA) was applied for all computational analyses.

\section{Results}

The flow chart representing the study group construction is presented in Figure $1 \mathrm{~A}$.
The sitting position was found in 41 (6.5\%) victims of cardiac arrest at EMS arrival. Among 20 patients with traumatic cardiac arrest, three (15\%) were sitting at EMS arrival, while among the rest of 614 patients, 38 (6.2\%) were sitting $(p=0.12)$ - Figure 1B.

The demographics, OHCA circumstances, and clinical data are presented in Table 1 and Table 2.

Patients found in a sitting position in comparison with those found lying were more frequently women: $46.3 \%$ vs. $30.6 \% p=0.036$, older: 74.0 (57.9-82.0) vs. 67.7 (54.9-75.8) $p=0.007$, less frequently given CPR by bystanders: $7.3 \%$ vs. $63.6 \% p<0.001$, the ROSC was found with similar frequency: $34.2 \%$ vs. $34.1 \% p=0.99$. Survival until hospital admission in the subgroup of prehospital ROSC patients earlier 
Table 1. Demographics and cardiac arrest circumstances

\begin{tabular}{lccc}
\hline & $\begin{array}{c}\text { Lying position } \\
\mathbf{n}=\mathbf{5 9 3}\end{array}$ & $\begin{array}{c}\text { Sitting position } \\
\mathbf{n = 4 1}\end{array}$ & P-value \\
\hline Males n (\%) & $410(69.4)$ & $22(53.7)$ & 0.036 \\
Age (years) - median (interquartile range) & $67.7(54.9-75.8)$ & $74.0(57.9-82.0)$ & 0.007 \\
Aetiology n (\%) & & & 0.26 \\
Medical & $502(86.3)$ & $34(82.9)$ & \\
Traumatic & $17(2.8)$ & $3(7.3)$ & 0.67 \\
Other & $64(11.0)$ & $4(9.8)$ & \\
Place n (\%) & & & \\
Home & $424(71.7)$ & $30(73.2)$ & $7(17.1)$ \\
Public place & $105(17.8)$ & $3(7.3)$ & $0(0)$ \\
Street/highway & $23(3.9)$ & $0(0)$ & \\
Workplace & $19(3.2)$ & $1(2.4)$ & \\
Outpatient clinic & $6(1.0)$ & $35(87.5)$ & 0.29 \\
Other & $14(2.4)$ & $3(7.3)$ & $<0.001$ \\
Witnessed n (\%) & $543(92.2)$ & $376(63.6)$ & \\
Bystander CPR n (\%) & 376 & \\
\hline
\end{tabular}

CPR - cardiopulmonary resuscitation

Table 2. Clinical and cardiac arrest description data

\begin{tabular}{|c|c|c|c|}
\hline & $\begin{array}{l}\text { Lying position } \\
\mathrm{n}=593\end{array}$ & $\begin{array}{c}\text { Sitting position } \\
n=41\end{array}$ & P-value \\
\hline Symptoms before CA n (\%) & $318(57.4)$ & $33(84.6)$ & $<0.001$ \\
\hline Dyspnoea n (\%) & $104(17.5)$ & $11(26.8)$ & 0.14 \\
\hline Chest pain $\mathrm{n}(\%)$ & $46(7.8)$ & $10(24.4)$ & 0.001 \\
\hline Weakness n (\%) & $70(11.8)$ & $9(22.0)$ & 0.057 \\
\hline Previous myocardial infarction n (\%) & $64(10.8)$ & $4(9.4)$ & 0.84 \\
\hline Diabetes n (\%) & $85(14.3)$ & $10(24.4)$ & 0.08 \\
\hline Stroke n (\%) & $26(4.4)$ & $1(2.4)$ & 0.55 \\
\hline $\mathrm{CABG} / \mathrm{PCl} \mathrm{n}(\%)$ & $30(5.1)$ & $1(2.4)$ & 0.45 \\
\hline $\mathrm{PM} / \mathrm{ICD} \mathrm{n}(\%)$ & $25(4.2)$ & $6(14.6)$ & 0.002 \\
\hline Time from emergency call to EMS arrival (min) & $8(8-11)$ & $8(8-9)$ & 0.50 \\
\hline $\begin{array}{l}\text { First recorded rhythm } \\
\text { VF/PVT } \\
\text { PEA } \\
\text { Asystole } \\
\text { Unknown }\end{array}$ & $\begin{array}{c}128(21.5) \\
62(10.4) \\
347(58.5) \\
56(9.6)\end{array}$ & $\begin{array}{c}7(17.0) \\
21(51.2) \\
8(19.5) \\
5(12.3)\end{array}$ & 0.30 \\
\hline Defibrillation n (\%) & $204(34.9)$ & $17(41.5)$ & 0.40 \\
\hline Epinephrine use $\mathrm{n}(\%)$ & $492(83 \%)$ & $36(87.8)$ & 0.42 \\
\hline Oropharyngeal tube $\mathrm{n}(\%)$ & $43(7.3)$ & $3(7.3)$ & 0.87 \\
\hline Airway mask n (\%) & $116(19.6)$ & $8(19.5)$ & \\
\hline Intubation n (\%) & $351(59.2)$ & $26(63.4)$ & \\
\hline Other or no data $\mathrm{n}(\%)$ & $83(14.0)$ & $4(9.8)$ & \\
\hline Venous access $n(\%)$ & $511(86.2)$ & $36(87.8)$ & 0.99 \\
\hline Intraosseus access only n (\%) & $3(0.51)$ & $0(0)$ & \\
\hline No data $n(\%)$ & 79 (13.3) & $5(12.2)$ & \\
\hline ROSC n (\%) & $200(34.1)$ & $14(34.2)$ & 0.99 \\
\hline Survival until hospital admission n (\%) & $151(25.4)$ & $7(17.1)$ & 0.23 \\
\hline Survival until hospital admission in ROSC group (\%) & 75.5 & 50.0 & 0.036 \\
\hline Survival until discharge $\mathrm{n}(\%)$ & $33(6.1)$ & $0(0)$ & 0.11 \\
\hline
\end{tabular}


Variable

Sex (male)

Previous ICD/PM

Chest pain before OHCA

Age (per 1 year increase)
OR (95\% Cl)

P-value

$0.568(0.283-1.141) \quad 0.11$

$3.675(1.250-10.802) \quad 0.02$

$5.175(2.286-11.714)<0.001$

$1.023(0.999-1.048) \quad 0.06$

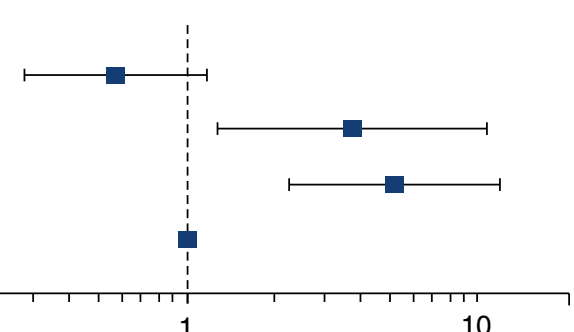

10

Figure 2. Independent factors associated with the sitting position of patients with OHCA on EMS arrival. Results of multivariable analysis. $\mathrm{Cl}$ - confidence interval; ICD/PM — implantable cardioverter-defibrillator/pacemaker; OHCA — out-of-hospital cardiac arrest; OR — odds ratio; P-value — probability value

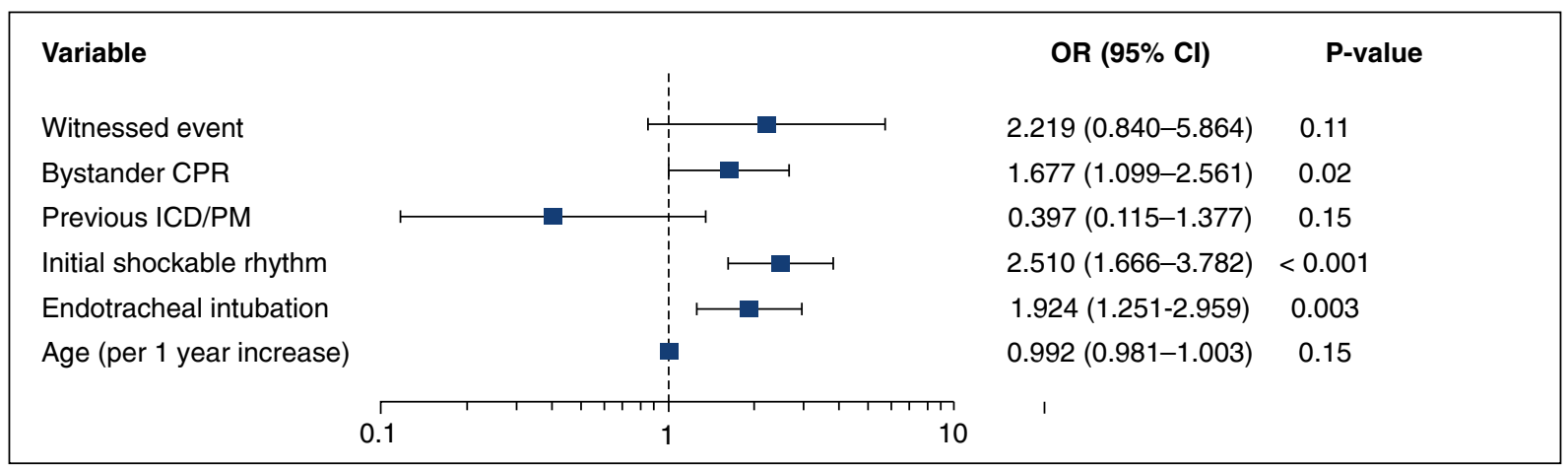

Figure 3. Independent predictors of the survival until hospital admission after OHCA. Results of multivariable analysis. $\mathrm{Cl}$ — confidence interval; CPR — cardiopulmonary resuscitation; ICD/PM — implantable cardioverter-defibrillator/pacemaker; P-value - probability value

found by EMS personnel in a sitting position was lower than those found in a lying position: $50 \%$ vs $75.5 \%$ $p=0.036$. None of the victims found arrested in a sitting position by EMS survived until hospital discharge, while survival until hospital discharge was found in $33(6.1 \%)$ victims found in a lying position: $p=0.11$.

The multivariable logistic regression analysis revealed that the sitting position was independently associated with chest pain before cardiac arrest and the presence of ICD or PM but not with sex or age (Fig. 2).

The univariable analysis showed that the sitting position was associated with female sex, older age, previously implanted ICD or PM, and chest pain before OHCA (Supplementary Materials, Table S2).

The independent predictors of survival until hospital admission included bystander CPR, shockable rhythm, and endotracheal intubation (Fig. 3). The witnesses of the event, ICD or PM, and age were not significantly or independently related to the transfer to the hospital with or without sustained ROSC.

The survival until hospital admission after OHCA was associated with the following factors in univari- able analysis: younger age, witnesses of the event, bystander CPR, initial shockable rhythm, endotracheal intubation, and borderline significantly with the lack of ICD or PM.

\section{Discussion}

The main finding of the study is that $6.5 \%$ of patients with OHCA were found in a sitting position at the arrival of Emergency Medical Services. The reason for maintaining the sitting position at EMS arrival was the lack of spontaneous fall of the victim and the lack of changing the victim's position by bystanders. No spontaneous fall may be related to the fastened seat belt in a vehicle or limited space around a victim that prevents adoption of a lower position or falling [16]. In fact, the sitting position occurred in $15 \%$ of patients with traumatic cardiac arrest where these factors frequently occur. Nonetheless, the traumatic injury as the cause of cardiac arrest concerned less than $10 \%$ of patients found by EMS in a sitting position. 
Reports regarding body position at EMS arrival to the arrested patient in the literature are scarce, and this was not the primary consideration of the available publications $[11,12,16]$. The victim's body position at EMS arrival was not included in the Utstein style reporting of cardiac arrest; therefore, the data were not systematically collected. However, some information is available from papers regarding the occurrence of cardiac arrest in toilets or circumstances of OHCA recorded on video [16].

Kiyohara et al. [12] reported that $4.6 \%$ of $\mathrm{OHCA}$ in Japan occurred in a toilet. The outcome of these events was poor, mainly because of delayed recognition. Hayashi et al. compared the percentage of cardiac arrests preceded by a given daily life activity with the estimated percentage of time spent performing it. Three kinds of activities were related to higher than expected incidence of OHCA, namely using a bath, which may be a special consideration in Japan due to the practice of taking very hot baths and their relationship to vasovagal syncope (VVS), using a toilet, walking or cycling for sport or strenuous work [17, 18]. Abe et al. [18] reported an increased incidence of OHCA due to drowning during bathing at home in Japan, particularly in the elderly. They presumed syncope due to the hot environment as the cause of drowning.

The second finding was that victims found in a sitting position were significantly older than those found lying and tended to be more frequently female. These differences in demographics suggest that patients found in a sitting position at ambulance arrival may differ from other victims in terms of the direct cause of cardiac arrest. Furthermore, in the multivariable analysis, the sitting position was independently associated with chest pain before cardiac arrest and the presence of ICD or PM but not with sex and age in the case of the sitting group. The victim's orthostatic posture may indicate the possibility that cardiac arrest could be provoked by central hypovolaemia due to orthostasis or reflex mechanisms. However, a sitting position in cardiac arrest may be due to limited space, immobilization by something preventing falling, or low centre of gravity, regardless of the cause of the cardiac arrest. In contrast, some patients, who faint, may fall and regain consciousness but have profound post-syncopal bradycardia and hypotension progressing to cardiac arrest in a lying position [19]. Thus, multiple factors play a part in the final position adopted by the victim of cardiac arrest.

The third finding is that only a small percentage received bystander CPR among arrested patients found in a sitting position. It could be caused by bystanders' inability to change the victim's position or lack of knowledge about its necessity. The latter explanation indicates poor knowledge of the CPR rules. CPR was rarely performed in arrested patients found by EMS in a sitting position. Moreover, it was proven that CPR is inefficient in this position. The environment in which the cardiac arrest may occur in any other than supine position is neurosurgery. The patients who undergo neurosurgery are placed in forced, non-supine positions to improve surgical access. The current guidelines indicate the necessity to put them into a supine position when they are in sitting or lateral positions. In case of a prone position, the patients are put in the supine position when chest compressions in the prone position are ineffective [20].

The fourth finding was that ROSC frequency in the sitting and lying groups was similar but did not lead to the same survival until hospital admission and discharge, which were lower for the group found in the sitting position. A similar ROSC frequency in sitting patients with cardiac arrest, compared with those found lying, despite adverse impact factors of older age and less frequent CPR, suggests the existence of other factors that may favorably influence their prognosis. These could include the pathogenesis of cardiac arrest.

The sitting position of victims may be an example of resuscitation in the head-up position, which was initially considered to improve the chances of ROSC [21]. However, recent findings have not confirmed this, although the head elevation in the lying position is believed to reduce cerebral edema [22]. ROSC depends mainly on the blood supply to the heart. This supply could be higher when blood supply to the brain is gravitationally reduced by a partial or complete upright position. However, ultimate survival depends on brain blood supply yielding better brain preservation, which is hindered in the sitting position.

A study assessing the usefulness of specific situational information such as the patient's posture in cardiac arrest did not help its recognition [23]. In cases of cardiac arrest in a sitting position, possibly imposed by the immediate surroundings, effective chest compressions require the patient to be lowered to a supine position [24]. The results of this study indicate the necessity to include advice into dispatcher education to encourage bystanders to change the victim's position to supine even before the arrival of EMS personnel. In the recording of events by EMS personnel, the victim's position when first found will prove helpful, and if the victim has remained in a sitting position, the advice should be to lower the patient to a supine position as quickly as possible.

The last finding of the study was that the independent predictors of survival until hospital admission included bystander CPR, shockable rhythm, and endotracheal intubation. This finding is consistent with other authors' reports and stresses the importance of the chain of survival. However, body position at EMS arrival may be an indicator of the lack of CPR or poor CPR quality, and therefore, may not be an independent factor related to poor outcomes.

The resuscitation protocols provide a basis for looking for factors related to CPR outcomes [25]. The inclusion of body position in such protocols may improve resuscitation effort outcomes. Changing the victim body 
position by a bystander should be considered as the first CPR manoeuvrer; however, the assessment of its significance requires further observational studies.

\section{Limitations}

The study's main limitation is the relatively small sample size, which might not allow reaching the statistical significance for survival until hospital discharge. The survival until hospital discharge is about $5 \%$ in the whole group, while it is $0 \%$ in the patients found in a sitting position. However, the study enabled the estimation of the frequency of the observed phenomenon of maintaining a sitting position by cardiac arrest victims at ambulance arrival and to plan further studies. The lack of some data is also a shortcoming of the study; however, this applied to less than $10 \%$ of the study group.

\section{Conclusions}

The sitting position of the out-of-hospital cardiac arrest victim at the arrival of Emergency Medical Services was recorded in $6.5 \%$ of patients in whom resuscitation attempts were undertaken. Victims found in a sitting position at the arrival of Emergency Medical Services frequently are older, more often female, experience chest pain, and have comorbidities than those found in a lying position, pointing out the need to seek the underlying mechanisms of cardiac arrest.

Victims found in a sitting position at the arrival of Emergency Medical Services received bystander CPR significantly less frequently and had similar chances for ROSC compared to those in supine position; however, those found sitting, who had ROSC, had slightly lower chances of survival until hospital admission.

\section{Conflict of interest: None.}

\section{References}

1. Cummins RO, Chamberlain DA, Abramson NS, et al. Recommended guidelines for uniform reporting of data from out-of-hospital cardiac arrest: the Utstein Style. A statement for health professionals from a task force of the American Heart Association, the European Resuscitation Council, the Heart and Stroke Foundation of Canada, and the Australian Resuscitation Council. Circulation. 1991; 84(2): 960-975, doi: 10.1161/01.cir.84.2.960, indexed in Pubmed: 1860248.

2. Berdowski J, Berg RA, Tijssen JGP, et al. Global incidences of out-of-hospital cardiac arrest and survival rates: Systematic review of 67 prospective studies. Resuscitation. 2010; 81(11): 1479-1487, doi: 10.1016/j.resuscitation.2010.08.006, indexed in Pubmed: 20828914.

3. Gräsner JT, Herlitz J, Koster RW, et al. Quality management in resuscitation--towards a European cardiac arrest registry (EuReCa). Resuscitation 2011; 82(8): 989-994, doi: 10.1016/j.resuscitation.2011.02.047, indexed in Pubmed: 21507548

4. Mozaffarian D, Benjamin EJ, Go AS, et al. Heart disease and stroke statistics--2015 update: a report from the American Heart Association. Circulation. 2015; 131(4): e29-322, doi: 10.1161/CIR.0000000000000152, indexed in Pubmed: 25520374
5. Gach D, Nowak JU, Krzych ŁJ. Epidemiology of out-of-hospital cardiac arrest in the Bielsko-Biala district: a 12-month analysis. Kardiol Pol. 2016; 74(10): 1180-1187, doi: 10.5603/KP.a2016.0086, indexed in Pubmed: 27221961

6. Nadolny K, Zyśko D, Obremska M, et al. Analysis of out-of-hospital cardiac arrest in Poland in a 1-year period: data from the POL-OHCA registry. Kardiol Pol. 2020; 78(5): 404-411, doi: 10.33963/KP.15241, indexed in Pubmed: 32191020.

7. D'Souza DH, Harish S, Rajesh M, et al. Rigor mortis in an unusual position: Forensic considerations. Int J Appl Basic Med Res. 2011; 1(2): 120-122, doi: 10.4103/2229-516X.91160, indexed in Pubmed: 23776792

8. Panhuyzen-Goedkoop NM, Wellens HJ, Piek JJ. Early recognition of sudden cardiac arrest in athletes during sports activity. Neth Heart $\mathrm{J}$. 2018; 26(1): 21-25, doi: 10.1007/s12471-017-1061-5, indexed in Pubmed: 29196876.

9. Bhatnagar V, Jinjil K, Dwivedi D, et al. Cardiopulmonary Resuscitation: Unusual Techniques for Unusual Situations. J Emerg Trauma Shock. 2018; 11(1): 31-37, doi: 10.4103/JETS.JETS 58 17, indexed in Pubmed: 29628666

10. Linderoth G, Møller TP, Folke F, et al. Medical dispatchers' perception of visual information in real out-of-hospital cardiac arrest: a qualitative interview study. Scand J Trauma Resusc Emerg Med. 2019; 27(1): 8, doi: 10.1186/s13049-018-0584-0, indexed in Pubmed: 30683139.

11. Freire-Tellado M, Pavón-Prieto MD, Fernández-López M, et al. Does the recovery position threaten cardiac arrest victim's safety assessment? Resuscitation. 2016; 105: e1, doi: 10.1016/j.resuscitation.2016.01.040, indexed in Pubmed: 27290992.

12. Kiyohara K, Nishiyama C, Kiguchi T, et al. Out-of-hospital cardiac arrests in the toilet in Japan: a population-based descriptive study. Acute Med Surg. 2018; 5(4): 369-373, doi: 10.1002/ams2.349, indexed in Pubmed: 30338084

13. Zyśko D, Fedorowski $A$, Nilsson $D$, et al. Tilt testing results are influenced by tilt protocol. Europace. 2016; 18(7): 1108-1112, doi: 10.1093/europace/euv262, indexed in Pubmed: 26443446.

14. Liu X, Asirvatham S, Kabell GG. Orthostatic increase in defibrillation threshold leading to defibrillation failure and prolonged cardiac arrest in a sitting position: Lessons from a patient's near-fatal experience. HeartRhythm Case Rep. 2016; 2(4): 313-317, doi: 10.1016/j.hrcr.2016.03.005, indexed in Pubmed: 28491699.

15. Nadolny K, Bujak K, Kucap M, et al. The Silesian Registry of Out-of-Hospital Cardiac Arrest: Study design and results of a three-month pilot study. Cardiol J. 2020; 27(5): 566-574, doi: 10.5603/CJ.a2018.0140, indexed in Pubmed: 30444257

16. Linderoth G, Hallas P, Lippert FK, et al. Challenges in out-of-hospital cardiac arrest - A study combining closed-circuit television (CCTV) and medical emergency calls. Resuscitation. 2015; 96: 317-322, doi: 10.1016/.j.resuscitation.2015.06.003, indexed in Pubmed: 26073272.

17. Hayashi $\mathrm{S}$, Toyoshima $\mathrm{H}$, Tanabe $\mathrm{N}$, et al. Activity immediately before the onset of non-fatal myocardial infarction and sudden cardiac death. Jpn Circ J. 1996; 60(12): 947-953, doi: 10.1253/jcj.60.947, indexed in Pubmed: 8996685

18. Abe H, Kohno R, Oginosawa Y. Characteristics of syncope in Japan and the Pacific rim. Prog Cardiovasc Dis. 2013; 55(4): 364-369, doi: 10.1016/j. pcad.2012.11.008, indexed in Pubmed: 23472772

19. Zyśko D, Fedorowski A, Sutton R. Cardiac arrest during recovery after tilt-induced vasodepressor syncope in a 76-year old man. Journal of Acute Medicine. 2016; 6(3): 67-69, doi: 10.1016/j.jacme.2016.05.002.

20. https://www.researchgate.net/publication/332820518_Management_of_ cardiac arrest during neurosurgery in adults (13.10.2020).

21. Ryu HHo, Moore JC, Yannopoulos D, et al. The Effect of Head Up Cardiopulmonary Resuscitation on Cerebral and Systemic Hemodynamics. Resuscitation. 2016; 102: 29-34, doi: 10.1016/j.resuscitation.2016.01.033, indexed in Pubmed: 26905388.

22. Park YJ, Hong KiJ, Shin SDo, et al. Worsened survival in the head-up tilt position cardiopulmonary resuscitation in a porcine cardiac arrest model. Clin Exp Emerg Med. 2019; 6(3): 250-256, doi: 10.15441/ceem.18.060, indexed in Pubmed: 31571441

23. Clawson J, Olola C, Heward A, et al. Ability of the medical priority dispatch system protocol to predict the acuity of "unknown probem" dispatch response levels. Prehosp Emerg Care. 2008; 12(3): 290-296, doi: 10.1080/10903120802100787, indexed in Pubmed: 18584494

24. Koster RW, Baubin MA, Bossaert LL, et al. European Resuscitation Council Guidelines for Resuscitation 2010 Section 2. Adult basic life support and use of automated external defibrillators. Resuscitation. 2010; 81(10): 1277-1292, doi: 10.1016/j.resuscitation.2010.08.009, indexed in Pubmed: 20956051

25. Jagosz A, Bursy D, Sobon A, et al. In-hospital sudden cardiac arrest protocol analysis. Kardiol Pol. 2018; 76(2): 376-380, doi: 10.5603/KPa2017.0209, indexed in Pubmed: 29131292. 